\title{
ABKÜRZUNGS- UND SIGLENVERZEICHNIS
}

\section{ABKÜRZUNGSVERZEICHNIS}

Abkürzungen gelten auch für die entsprechenden Flexionsformen. Adjektive auf -lich und -isch werden in der Regel abgekürzt.

\begin{tabular}{|c|c|c|c|}
\hline Abb. & Abbildung(en) & Bibl. & Bibliothek(en), Bibliot(h)eca, \\
\hline Abdr. & Abdruck & & Bibliothèque \\
\hline Abh. & Abhandlung(en) & bibliogr. & bibliographisch \\
\hline Abt. & Abteilung(en) & Bibliogr. & Bibliographie(n) \\
\hline ahd. & althochdeutsch & biogr. & biographisch \\
\hline Akad. & Akademie(n) & Biogr. & Biographie(n) \\
\hline allg. & allgemein & Bl. & Blatt, Blätter \\
\hline Anh. & Anhang & $\mathrm{BR}$ & Bayerischer Rundfunk \\
\hline Anm. & Anmerkung(en) & Briefw. & Briefwechsel \\
\hline Ann. & $\begin{array}{l}\text { Annalen, Annales, Annals, } \\
\text { Annali }\end{array}$ & BSB & $\begin{array}{l}\text { Bayerische Staatsbibliothek, } \\
\text { München }\end{array}$ \\
\hline anon. & anonym & Bull. & Bulletin \\
\hline Anthol. & Anthologie(n) & Burl. & Burleske(n) \\
\hline Anz. & Anzeiger, Anzeigen & & \\
\hline a. o. Prof. & außerordentl. Prof. & $\mathrm{CD}$ & Compact Disc \\
\hline $\begin{array}{l}\text { apl. Prof. } \\
\text { Arch. }\end{array}$ & $\begin{array}{l}\text { außerplanmäßige(r) Prof. } \\
\text { Archiv }\end{array}$ & $\mathrm{CDU}$ & $\begin{array}{l}\text { Christlich Demokratische } \\
\text { Union }\end{array}$ \\
\hline ARD & $\begin{array}{l}\text { Arbeitsgemeinschaft der } \\
\text { öffentlich-rechtlichen Rund- } \\
\text { funkanstalten der Bundes- } \\
\text { republik Deutschland }\end{array}$ & $\begin{array}{l}\text { Chron. } \\
\text { Cod. } \\
\text { CSU }\end{array}$ & $\begin{array}{l}\text { Chronik(en) } \\
\text { Codex, Codices } \\
\text { Christlich-Soziale Union in } \\
\text { Bayern }\end{array}$ \\
\hline AT & Altes Testament & Cty. & County \\
\hline Auff. & Aufführung(en) & CVP & Christlichdemokratische \\
\hline Aufl. & Auflage(n) & & Volkspartei \\
\hline Aufs. & Aufsatz, Aufsätze & & \\
\hline Aufz. & Aufzeichnung(en) & D. & Drama \\
\hline Ausg. & Ausgabe(n) & d. $\ddot{A}$. & der (die) Ältere \\
\hline ausgew. & ausgewählt & Darst. & Darstellung(en) \\
\hline Ausw. & Auswahl & dass. & dasselbe \\
\hline Ausz. & Auszug, Auszüge & dems. & demselben \\
\hline autobiogr. & autobiographisch & Dép. & Département \\
\hline Autobiogr. & Autobiographie(n) & ders. & derselbe \\
\hline Ball. & Ballade(n) & DFG & $\begin{array}{l}\text { Deutsche Forschungs- } \\
\text { gemeinschaft }\end{array}$ \\
\hline $\mathrm{BBC}$ & $\begin{array}{l}\text { British Broadcasting } \\
\text { Corporation }\end{array}$ & $\begin{array}{l}\text { Dg. } \\
\text { d. Gr. }\end{array}$ & $\begin{array}{l}\text { Dichtung(en) } \\
\text { der (die) Große }\end{array}$ \\
\hline Bd., Bde., Bdn. & Band, Bände, Bänden & d. i. & das ist \\
\hline bearb., Bearb. & $\begin{array}{l}\text { bearbeitet, Bearbeiter(in), } \\
\text { Bearbeitung }\end{array}$ & $\begin{array}{l}\text { Dial. } \\
\text { dies. }\end{array}$ & $\begin{array}{l}\operatorname{Dialog}(\mathrm{e}) \\
\text { dieselbe(n) }\end{array}$ \\
\hline begr. & begründet & Diss. & Dissertation \\
\hline Beih. & Beiheft(e) & d. J. & der (die) Jüngere \\
\hline Beitr. & Beitrag, Beiträge & DLA & Deutsches Literaturarchiv/ \\
\hline Bem. & Bemerkung(en) & & Schiller-Nationalmus., \\
\hline Ber. & Bericht $(e)$ & & Marbach \\
\hline bes. & besonders & DLF & Deutschlandfunk \\
\hline Bez. & Bezirk & DNB & Deutsche Nationalbibl. \\
\hline
\end{tabular}




\begin{tabular}{|c|c|c|c|}
\hline Doz. & Dozent(in) & FS & Festschrift, Festgabe \\
\hline Dr. & Doktor & FU & Freie Univ. \\
\hline Drehb. & Drehbuch & & \\
\hline $\begin{array}{l}\text { dt. } \\
\text { durchges. }\end{array}$ & $\begin{array}{l}\text { deutsch } \\
\text { durchgesehen(e) }\end{array}$ & GDZ & $\begin{array}{l}\text { Göttinger Digitalisierungs- } \\
\text { zentrum }\end{array}$ \\
\hline & & geb. & geborene \\
\hline ebd. & ebenda & Ged. & Gedicht $(\mathrm{e})$ \\
\hline ed. & editio, edidit, ediert v., & gedr. & gedruckt \\
\hline & edited by & gegr. & gegründet \\
\hline ehem. & ehemalig(er), ehemals & gem. & gemeinsam \\
\hline eig. & eigentlich & gen. & genannt \\
\hline Einf. & Einführung(en) & ges. & gesammelt(e) \\
\hline eingel. & eingeleitet & Gesch. & Geschichte(n) \\
\hline Einl. & Einleitung(en) & Gesellsch. & Gesellschaft \\
\hline enth. & enthält, enthalten(d) & Ggw. & Gegenwart \\
\hline Ep. & Epos, Epen & GSA & Goethe-Schiller-Arch., \\
\hline Epigr. & Epigramm(e) & & Weimar \\
\hline & ergänzt & GSt.arch. & Geheimes Staatsarch. \\
\hline ErgBd., ErgBde. & Ergänzungsband, & & \\
\hline & Ergänzungsbände & H. & Heft(e) \\
\hline $\mathrm{ErgH}$ & Ergänzungsheft(e) & HAAB & Herzogin Anna Amalia Bibl \\
\hline Erinn. & Erinnerung(en) & $\mathrm{HAB}$ & Herzog August-Bibl., \\
\hline erl., Erl. & erläutert, Erläuterung(en) & & Wolfenbüttel \\
\hline ersch. & erschienen & Habil. & Habilitation \\
\hline erw. & erweitert & h.c. & honoris causa \\
\hline Erz. & Erzähler, Erzählung(en) & hd. & hochdeutsch \\
\hline Ess. & Essay(s) & Hdb. & Handbuch, Handbücher \\
\hline ETH & Eidgenössische Technische & hebr. & hebräisch \\
\hline evang. & $\begin{array}{l}\text { Hochschule } \\
\text { evangelisch }\end{array}$ & hg., Hg. & $\begin{array}{l}\text { herausgegeben (von), } \\
\text { Herausgeber(in) }\end{array}$ \\
\hline Ex. & Exemplar & $\begin{array}{l}\text { hist. } \\
\text { hl. }\end{array}$ & $\begin{array}{l}\text { historisch } \\
\text { heilig }\end{array}$ \\
\hline f., ff. & folgende Seite(n), folgende & Hörsp. & Hörspiel \\
\hline & $\begin{array}{l}\text { Spalte(n), folgendes (folgen- } \\
\text { de) Jahr(e) }\end{array}$ & $\begin{array}{l}\text { HR } \\
\text { hs., Hs., Hss. }\end{array}$ & $\begin{array}{l}\text { Hessischer Rundfunk } \\
\text { handschriftlich, Handschrift, }\end{array}$ \\
\hline F. & Folge & & Handschriften \\
\hline Facs. & Facsimile, Faksimile & $\mathrm{HU}$ & Humboldt-Univ. \\
\hline Fak. & Fakultät(en) & & \\
\hline Fass. & Fassung & illustr., Illustr. & illustriert, Illustration(en) \\
\hline FDH & $\begin{array}{l}\text { Freies Dt. Hochstift - } \\
\text { Frankfurter Goethemus., } \\
\text { Frankfurt/M. }\end{array}$ & $\begin{array}{l}\text { insbes. } \\
\text { in Verb. mit } \\
\text { in Vorb. }\end{array}$ & $\begin{array}{l}\text { insbesondere } \\
\text { in Verbindung mit } \\
\text { in Vorbereitung }\end{array}$ \\
\hline FDP & Freie Demokratische Partei & Inst. & Institut(e) \\
\hline FPÖ & $\begin{array}{l}\text { Freiheitliche Partei } \\
\text { Österreichs }\end{array}$ & $\begin{array}{l}\text { Interpr. } \\
\text { ital. }\end{array}$ & $\begin{array}{l}\text { Interpretation(en) } \\
\text { italienisch }\end{array}$ \\
\hline Feuill. & Feuilleton(s) & & \\
\hline $\begin{array}{l}\text { fol. } \\
\text { Forsch. }\end{array}$ & $\begin{array}{l}\text { folio } \\
\text { Forschung(en) }\end{array}$ & $\begin{array}{l}\text { Jb. } \\
\text { Jber. }\end{array}$ & $\begin{array}{l}\text { Jahrbuch, Jahrbücher } \\
\text { Jahresbericht(e) }\end{array}$ \\
\hline $\begin{array}{l}\text { Forscn. } \\
\text { Forts. }\end{array}$ & $\begin{array}{l}\text { Forschung(en) } \\
\text { Fortsetzung(en) }\end{array}$ & $\begin{array}{l}\text { Jer. } \\
\text { Jg. }\end{array}$ & $\begin{array}{l}\text { Janresberic nt(e) } \\
\text { Jahrgang, Jahrgänge }\end{array}$ \\
\hline fragm., Fragm. & fragmentarisch, Fragment(e) & Jgdb. & Jugendbuch \\
\hline Frfr. & Freifrau & Jh. & Jahrhundert(e) \\
\hline Frhr. & Freiherr & & \\
\hline frz. & französisch & Kap. & Kapitel \\
\hline
\end{tabular}




\begin{tabular}{|c|c|c|c|}
\hline Kat. & Katalog(e) & Nachw. & Nachwort \\
\hline kath. & katholisch & nd. & niederdeutsch \\
\hline Kdb. & Kinderbuch & NDR & Norddeutscher Rundfunk \\
\hline K1. & Klasse & nds. & niedersächsisch \\
\hline Kom. & Komödie(n) & Neudr. & Neudruck(e) \\
\hline komm., Komm. & $\begin{array}{l}\text { kommentiert(e), } \\
\text { Kommentar(e) }\end{array}$ & NF & Neue Folge \\
\hline \multirow[t]{2}{*}{ KPD } & Kommunistische Partei & nlat. & $\begin{array}{l}\text { neulateinisch } \\
\text { nat }\end{array}$ \\
\hline & Deutschlands & Nov. & Novelle(n) \\
\hline \multirow[t]{2}{*}{ KPÖ } & Kommunistische Partei & Nr. & Nummer \\
\hline & Österreichs & NS & Neue Serie, Nova Series, \\
\hline $\mathrm{Kr}$. & Kreis & & New Series, Nouvelle Série, \\
\hline \multirow{2}{*}{ Kt. } & Kanton & & Nuova Seria \\
\hline & lateinisch & NSDAP & $\begin{array}{l}\text { Nationalsozialistische Deut- } \\
\text { sche Arbeiterpartei }\end{array}$ \\
\hline LB & Landesbibl. & NT & Neues Testament \\
\hline Lb., Lbb. & Lebensbild, Lebensbilder & NWDR & Nordwestdeutscher \\
\hline Leg. & Legende $(n)$ & & Rundfunk \\
\hline Lex. & Lexikon, Lexika & & \\
\hline Lfg. & Lieferung & ÖNB & Österreichische Nationalbibl \\
\hline Libr. & Libretto, Libretti & öst. & österreichisch \\
\hline lic. & licentiatus & ÖVP & Österreichische Volkspartei \\
\hline lit., Lit. & literarisch, Literatur(en) & o. J. & ohne Jahr \\
\hline Lkr. & Landkreis & op. & opus \\
\hline LP & Langspielplatte & o. Prof. & ordentl. Prof. \\
\hline Lsp. & Lustspiel & ORB & Ostdeutscher Rundfunk \\
\hline luth. & lutherisch & ORF & Österreichischer Rundfunk \\
\hline \multirow[t]{2}{*}{ M. A. } & Magister Artium, Master of & P. & Prosa \\
\hline & Arts & Pap. & Papier \\
\hline \multirow{2}{*}{$\begin{array}{l}\text { MA, ma. } \\
\text { masch. }\end{array}$} & Mittelalter, mittelalterlich & Par. & Parodie(n) \\
\hline & maschinenschriftlich & Perg. & Pergament \\
\hline Mbl. & Monatsblatt, Monatsblätter & $\mathrm{PH}$ & Pädagog. Hochschule \\
\hline MDR & Mitteldeutscher Rundfunk & Philol. & Philologie \\
\hline mhd. & mittelhochdeutsch & philos., Philos. & philosophisch, Philosophie \\
\hline Mitarb. & Mitarbeit(er, -erin) & Präs. & Präses, Präsident(in) \\
\hline Mitgl. & Mitglied(er) & Prof. & Professor(in) \\
\hline Mitt. & Mitteilung(en) & Progr. & $\operatorname{Programm}(\mathrm{e})$ \\
\hline Mitw. & Mitwirkung & Prov. & Provinz \\
\hline mlat. & mittellateinisch & Ps. & Pseudonym(e) \\
\hline mnd. & mittelniederdeutsch & Publ. & Publikation(en), \\
\hline $\begin{array}{l}\text { m.n.e. } \\
\text { mnl. }\end{array}$ & $\begin{array}{l}\text { mehr nicht erschienen } \\
\text { mittelniederländisch }\end{array}$ & & Publication(s) \\
\hline Monogr. & Monographie(n) & Qschr. & Quartalschrift(en) \\
\hline Ms., Mss. & Manuskript, Manuskripte & & \\
\hline Mschr. & Monatsschrift & R. & Reihe(n) \\
\hline Msp. & Märchenspiel & RB & Radio Bremen \\
\hline \multirow[t]{2}{*}{ Mus. } & Museum & $\mathrm{RBB}$ & Rundfunk Berlin \\
\hline & & & Brandenburg \\
\hline NA & Neuauflage & $\mathrm{rd}$ & rund \\
\hline Nachdr. & Nachdruck(e) & red., Red. & redigiert, Redaktion, \\
\hline Nachr. & Nachricht(en) & & Redakteur(in) \\
\hline Nachtr. & Nachtrag, Nachträge & Reg. & Register \\
\hline
\end{tabular}




\begin{tabular}{|c|c|c|c|}
\hline Rel., rel. & Religion, religiös & Sz. & Szene(n) \\
\hline Rep. & Reportage $(\mathrm{n})$ & & \\
\hline Resp. & Respondent(en) & $\mathrm{Tb}$. & Taschenbuch \\
\hline rev. & revidiert & $\mathrm{TH}$ & Techn. Hochschule \\
\hline Rev. & Revue, Review & Theol. & Theologie \\
\hline RIAS & $\begin{array}{l}\text { Rundfunk im amerikani- } \\
\text { schen Sektor }\end{array}$ & $\begin{array}{l}\text { Tl., Tle., Tln. } \\
\text { Trag. }\end{array}$ & $\begin{array}{l}\text { Teil, Teile, Teilen } \\
\text { Tragödie(n) }\end{array}$ \\
\hline Rom. & Roman $(e)$ & Trauersp. & Trauerspiel(e) \\
\hline Rs. & Rundschau & Tril. & Trilogie \\
\hline RSB & Radio Saarbrücken & $\begin{array}{l}\text { Td. } \\
\text { TU }\end{array}$ & $\begin{array}{l}\text { Tausend } \\
\text { Techn. Univ. }\end{array}$ \\
\hline s. & siehe & tw. & teilweise \\
\hline S. & Seite(n) & & \\
\hline Sachb. & Sachbuch & u. & und \\
\hline $\mathrm{Sb}$. & Sitzungsbericht(e) & u. a. & und andere, unter anderem \\
\hline SB & Staatsbibl. & u. ä. & und ähnliche(s) \\
\hline SBPK & $\begin{array}{l}\text { Staatsbibl. zu Berlin Preuß. } \\
\text { Kulturbesitz }\end{array}$ & $\begin{array}{l}\text { UB } \\
\text { u. d. T. }\end{array}$ & $\begin{array}{l}\text { Univ.bibl. } \\
\text { unter dem Titel }\end{array}$ \\
\hline Schausp. & Schauspiel & überarb. & überarbeitet(e) \\
\hline Schr. & Schrift(en) & überl., Überl. & überliefert, Überlieferung \\
\hline Schriftst. & Schriftsteller(in) & übers., Übers. & übersetzt, Übersetzer(in), \\
\hline Schw. & Schwank, Schwänke & & Übersetzung(en) \\
\hline schweiz. & schweizerisch & übertr., Übertr. & übertragen, Übertragung(en) \\
\hline SDR & Süddeutscher Rundfunk & ULB & Univ.- u. Landesbibl. \\
\hline SED & Sozialistische Einheitspartei & unbek. & unbekannt \\
\hline & Deutschlands & ungedr. & ungedruckt \\
\hline sep. & separat & Univ. & Universität(en), Université, \\
\hline SFB & Sender Freies Berlin & & University \\
\hline Slg. & Sammlung(en) & Unters. & Untersuchung(en) \\
\hline SLUB & $\begin{array}{l}\text { Sächs. LB - Staats- und } \\
\text { Univ.bibl. Dresden }\end{array}$ & $\begin{array}{l}\text { unvollst. } \\
\text { u. ö. }\end{array}$ & $\begin{array}{l}\text { unvollständig } \\
\text { u. öfter }\end{array}$ \\
\hline sog. & sogenannt & Urauff. & Uraufführung \\
\hline Son. & Sonett(e) & urspr. & ursprünglich \\
\hline Sp. & Spiel(e) & USPD & Unabhängige Sozialdemo- \\
\hline SPD & $\begin{array}{l}\text { Sozialdemokratische Partei } \\
\text { Deutschlands }\end{array}$ & usw. & $\begin{array}{l}\text { kratische Partei Deutschlands } \\
\text { und so weiter }\end{array}$ \\
\hline SPÖ & Sozialdemokratische Partei & & \\
\hline & Österreichs & v. & von \\
\hline SR & Saarländischer Rundfunk & V., VV. & Vers, Verse \\
\hline SRG & Schweizer Rundspruch- & $\begin{array}{l}\text { v. a. } \\
\text { Vhdo }\end{array}$ & $\begin{array}{l}\text { vor allem } \\
\text { Verbindung }\end{array}$ \\
\hline SStB & Staats- u. Stadtbibl. & Ver. & Verein(e), Vereinigung(en) \\
\hline St. & Sankt, Saint; Stück(e) & verb. & verbessert \\
\hline StB & Stadtbibl. & Verf. & Verfasser(in) \\
\hline StLB & Stadt- u. Landesbibl. & verh. & verheiratet \\
\hline Str. & Strophe(n) & verm. & vermehrt \\
\hline StUB & Stadt- u. Univ.bibl. & veröff., Veröff. & veröffentlicht, \\
\hline Stud. & Studium, Studie(n) & & Veröffentlichung(en) \\
\hline SUB & Staats- u. Univ.bibl. & vers. & versehen \\
\hline Suppl. & Supplement(e) & versch. & verschieden $(e,-e s)$ \\
\hline SVP & Schweizerische Volkspartei & verw. & verwitwet \\
\hline SWF & Südwestfunk & Verz. & Verzeichnis(se) \\
\hline SWR & Südwestrundfunk & Vgh. & Vergangenheit \\
\hline
\end{tabular}




$\begin{array}{llll}\text { vgl. } & \text { vergleiche } & \text { zahlr. } & \text { zahlreiche } \\ \text { Vjs. } & \text { Vierteljahresschrift } & \text { z. B. } & \text { zum Beispiel } \\ \text { Volksk. } & \text { Volkskunde } & \text { ZB } & \text { Zentralbibl. } \\ \text { Volksst. } & \text { Volksstück } & \text { Zeichn. } & \text { Zeichnung(en) } \\ \text { vollst. } & \text { vollständig } & \text { ZLB } & \text { Zentral- und } \\ \text { Vorw. } & \text { Vorwort } & & \text { Landesbibliothek } \\ & & \text { Zs. } & \text { Zeitschrift(en) } \\ \text { wahrsch. } & \text { wahrscheinlich } & \text { Ztg. } & \text { Zeitung(en) } \\ \text { Wb. } & \text { Wörterbuch } & \text { zus. } & \text { zusammen } \\ \text { WDR } & \text { Westdeutscher Rundfunk } & \text { zus.gest. } & \text { zusammengestellt } \\ \text { wiss., Wiss, } & \text { wissenschaftlich, } & \text { zw. } & \text { zwischen } \\ \text { Ws. } & \text { Wissenschaft(en) } & \text { z. Z. } & \text { zur Zeit }\end{array}$

\section{ABKÜRZUNGEN GEOGRAPHISCHER NAMEN}

$\mathrm{Br}$

Burgenl.

Dtl.

Erzgeb.

Frankfurt/M.

Frankfurt/O.

Friesl.

Breisgau
Burgenland
Deutschland
Erzgebirge
Frankfurt am Main
Frankfurt an der
Oder
Friesland
Holstein
Kurland
Livland
Mecklenburg
Niederbayern
Niedersachsen
Oberbayern
Österreich
Pommern
Preußen

Rhld.

Rheinland

Schles.

Schlesien

Siebenb.

Siebenbürgen

Thür.

Thüringen

Ts.

Taunus

Vogtl.

Westf.

Vogtland

Württ.

Westfalen

Holst.

Kurl.

USA-Bundesstaaten:

Livl.

Mecklenb.

$\mathrm{Ndb}$.

Nds.

Obb.

Öst.

Pomm.

Pr.

Conn.

Ill.

Württemberg

Kalif.

Mass.

Mich.

N. Y.

Wash.

Wisc.

Connecticut

Illinois

Kalifornien

Massachusetts

Michigan

New York (Staat)

Washington

Wisconsin

\section{SIGLENVERZEICHNIS}

$\mathrm{AAB}$

AAG

AAH

AAM
Abh. der Dt. (ab I946; bis

dahin Preuß.) Akad. der

Wiss.. zu Berlin. Phil.-hist. $\mathrm{Kl}$., I804 ff.

Abh. der Königl. Gesellsch. der Wiss., Göttingen

Abh. der Heidelberger

Akad. der Wiss. Phil.-hist.

K1., I9I3 ff.

Abh. der Bayer. Akad. der

Wiss. Phil.-hist. Kl., I833 ff., I9IO ff.
ABäG

$\mathrm{ABnG}$

ADB

Adelung
Amsterdamer Beitr. zur älteren Germanistik, Amsterdam I972 ff.

Amsterdamer Beitr. zur neueren Germanistik, Amsterdam I972 ff.

Allg. Dt. Biogr., 55 Bde., Reg.-Bd., I875-I9I2

Allg. Gelehrten-Lex. v. C. G. Jöcher, Forts. v. J. C. Adelung $\mathrm{u}$. H. W. Rotermund, 7 Bde., I784-I879 
AfdA

AfK

AG

$\mathrm{AH}$

$\operatorname{AION}(\mathrm{T})$

AKL

Albrecht-Dahlke

Archiv

ARG

ARW

Aufriß

AUMLA

Autorenlex.

$\mathrm{BA}$
Anz. für dt. Altertum u. dt. Baader

Lit., I876-1989

Arch. für Kulturgesch., $1903 \mathrm{ff}$.

Acta Germanica. Kapstadt I966 ff.

Analecta Hymnica Medii Aevi (hg. C. Blume, G. M. Dreves [u. H. M. Bannister]) 55 Bde., I886-I922 (Nachdr. I96I; Reg., hg. M. Lütolf, Bd. I/I, I/2, II, I978)

Istituto Universitario Orientale. Annali. Sezione Germanica. Studi Tedeschi, Neapel I958 ff.

Allgemeines Künstlerlex.

Die Bildenden Künstler aller Zeiten u. Völker, I992 ff.

Internationale Bibliogr. zur Gesch. der dt. Lit. v. den Anfängen bis zur Ggw. unter Leitung u. Gesamtred. v. G. Albrecht u. G. Dahlke, 4 Tle., I969-84

Arch. für das Stud. der neueren Sprachen u. Literaturen, I846 ff.

Arch. für Reformationsgesch., I903 ff.

Arch. für Rel.wiss., I898-I94I/ 42

Dt. Philol. im Aufriß, hg.

W. Stammler, Nachdr. der 2., überarb. Aufl., 3 Bde. u. I Reg.bd., I978

AUMLA, Journal of the Australasian Universities Language and Literature Association, Christchurch I953 ff.

Autorenlex. dt.sprachiger Lit. des 20. Jh. (überarb. u. erw. Neuausg., hg. M. Brauneck) I995

Books Abroad, I-50 Norman/Oklahoma 1927-76 (ab 5I, I977, u.d.T.: World Literary Today)

$\mathrm{BB}$

BDL

BWG
C. A. Baader, Lex. verstorbener bayer. Schriftst. des I8. u. I9. Jh., 2 Bde., I $824 / 25$

Bartsch-Golther

K. Bartsch, Dt. Liederdichter des I2. bis I4. Jh. Eine Ausw., I864 (4. Aufl., besorgt v. W. Golther, I9OI; Nachdr. 1966)

Bayer. Bibl. Texte aus zwölf Jh., hg. H. Pörnbacher u. B. Hubensteiner, 5 Bde., I978-90

Bibl. der Dt. Lit.

Mikrofiche-Gesamtausg. nach Angaben des Taschengoedeke (bearb. A. Frey) I995 (2., überarb. u. erw. Ausg. u. Suppl. I, I999; Suppl. 2, 2002-05)

Biogr.-Bibliogr. Kirchenlex.

Biogr.-Bibliogr. Kirchenlex., bearb. u. hg. F. W. Bautz, fortgefuihrt v. T. Bautz, I975 ff.

Biogr. Jb.

Biogr. Jb. u. Dt. Nekrolog, hg. A. Bettelheim, I8 Bde., I897-I9I7, 2 Reg.bde., I908 u. 1973

Börsenbl. Leipzig Börsenbl. für den Dt. Buchhandel, hg. vom Börsenverein der Dt. Buchhändler zu Leipzig, I834 ff. (I945-I990: Zusatz «Leipzig»)

Börsenbl. Frankfurt Börsenbl. für den Dt. Buchhandel, Frankfurter Ausg. I945-I990

de Boor-Newald Gesch. der dt. Lit. v. den Anfängen bis zur Gegenwart, hg. H. de Boor u. R. Newald, I949 ff.

Braune-Ebbinghaus Ahd. Lesebuch. Zus.gest. u. mit einem Wb. vers. v. W. Braune, fortgeführt v. E. A. Ebbinghaus, I7. Aufl., I994

Brunhölzl F. Brunhölzl, Gesch. der lat. Lit. des MA, 2 Bde., I975 u. I992

Biogr. Wb. zur dt. Gesch., 2. Aufl., hg. K. Bosl, G. Franz u. H. H. Hofmann, 3 Bde., 1973-75 
CineGraph

CL

CollGerm.

Cramer

Daphnis

DB

DBE

Denecke-Brandis

Dict. Spir.

DL

DLE

DLZ

DMA

DNL

DR

DSL

Dt. biogr. Jb.
CineGraph. Lex. zum dt.sprachigen Film, hg. H.-B. Bock, I984 ff.

Comparative Literature, Eugene/Oregon I949 ff. Colloquia Germanica, I967 ff.

T. Cramer, Die kleineren Liederdichter des I4. u. I5. Jh., 4 Bde., I977-85

Daphnis, Zs. für Mittlere Dt. Lit., I972 ff.

Dt. Bücher, Amsterdam I97I ff.

Dt. Biogr. Enzyklopädie, hg. W. Killy u. R. Vierhaus, I3 Bde., I995-2003; 2., überarb. u. erw. Ausg., hg. R. Vierhaus, I2 Bde., 2005-08

Die Nachläse in den Bibl. der Bundesrepublik Dtl., bearb. v. L. Denecke; 2., Aufl., völlig neu bearb. v. T. Brandis, I98I

Dictionnaire de spiritualité ascétique et mystique. Doctrine et histoire. Fondé par M. Viller ... I7 Bde., Paris I937-95

Die dt. Lit. [...] Texte u. Zeugnisse, hg. W. Killy, 7 Bde., I963-78 (Nachdr. I988)

Dt. Lit. Slg. lit. Kunst- u. Kulturdenkmäler in Entwicklungsreihen, hg. $\mathrm{H}$.

Kindermann, I928-50

(Nachdr. I964-70)

Dt. Lit.-Ztg., I880 ff.

Dictionary of the Middle

Ages, hg. J. R. Strayer,

I3 Bde., New York

I982-89; Suppl. I, 2004

Dt. National-Lit., hg. J.

Kürschner, I882-99

Dt. Rundschau, I874 ff.

Die Schöne Lit., I924 ff.

Dt. biogr. Jb., hg. H. Christern, II Bde., I925-32, Reg.bd. I986
DU

Dünnhaupt

DVjs

EG

Ehrismann

Ersch-Gruber

Euph.

FA

Fabula

$\mathrm{FdF}$

FH

Flood, Poets

Laureate

Frels

GA

GermWrat

GGA

GLL
Der Deutschunterricht, I949 ff.

G. Dünnhaupt, Personalbibliogr. zu den Drucken des Barock. 2., verb. u. wesentl. verm. Aufl. des Bibliogr. Hdb. der Barocklit., 6 Bde., I990-93

Dt. Vjs. für Lit.-wiss. u Geistesgesch., I923-44, I949 ff.

Etudes germaniques, Paris I946 ff.

G. Ehrismann, Gesch. der dt. Lit. bis zum Ausgang des MA, 4 Bde., I9I8-35 Allg. Encyclopädie der Wiss. u. Künste, begr. v. J. S. Ersch u. J. G. Gruber, I67 Bde., I8I8-89, unvollständiger Nachdr. I969-92 Euphorion. Zs. für Lit.gesch, I894 ff.

Frankfurter Anthol., hg. M. Reich-Ranicki, I976 ff.

Fabula. Zs. für Erzählforsch., I960 ff.

C. Faber du Faur, German Baroque Literature, New Haven, Bd. I, I958, Bd. 2, I969

Frankfurter H., Zs. für Kultur u. Politik, I946 ff.

J. L. Flood, Poets Laureate in the Holy Roman Empire. A Bio-bibliograpical Handbook, 4 Bde., 2006 W. Frels, Dt. Dichterhss. I400-I900, I934

Gesammtabenteuer, hg. F. H. v. der Hagen, 3 Bde., I850 (Neudr. I96I; NA des I. Bd. I968)

Germanica Wratislaviensia, Breslau I957 ff.

Göttingsche Gelehrte Anz., I739 ff.

German Life and Letters, Oxford $1936 \mathrm{ff}$. 
Goedeke

Hall-Renner

HBLS

Hdb. Editionen

Hdb. Emigration

$\mathrm{HdG}$

Heiduk

Hist. Wb.

Rhetorik
K. Goedeke, Grundriß zur Gesch. der dt. Dg. aus den Quellen, 2. Aufl. I884 ff.; $\mathrm{IV} / \mathrm{I}-5$ 3. Aufl. I9Io ff.; NF I955 ff.

M. G. Hall u. G. Renner, Hdb. der Nachlässe u. Slg. öst. Autoren, I992; 2., neu bearb. u. erw. Aufl. I995

Hamberger/Meusel G. C. Hamberger, J. G. Meusel, Das gelehrte Teutschland oder Lex. der jetzt lebenden teutschen Schrift., 5., verm. u. verb. Ausg., 23 Bde., I796-I834 (Nachdr. I965/66; Reg.bd. I979)

Hist.-Biogr. Lex. der

Schweiz, 7 Bde., I92I-34

$\mathrm{Hdb}$. der Editionen.

Dt.sprachige Schriftst. vom Ausgang des I5. Jh. bis zur Ggw., bearb. v. W. Hagen, I. Jensen, E. u. H. Nahler, ${ }^{2}$ I98 I

Biogr. Hdb. der dt.sprachigen Emigration nach I933. Hg. Inst. für Zeitgesch., München, u Research Foundation of Jewish Emigration, Inc., New York, 3 Bde., I980-83 $\mathrm{Hdb}$. der dt. Gegenwartslit., 3 Bde., hg. H. Kunisch u. a., ${ }^{2}$ I969/70

F. Heiduk, Oberschles. Lit.Lex. Biogr.-bibliogr. Hdb., 3 Bde., I990-2000 Hist. Wb. der Rhetorik, hg. G. Ueding, I992 ff.
HMS

HZ

IASL

IG

Inscape

KLG

HLS

Hist. Lex. der Schweiz, hg. Stiftung Hist. Lex. der Schweiz, 2002 ff. (auch online, e-HLS)

Minnesinger. Ges. u. hg. v. F. H. v. der Hagen, 7 Tle. in 3 Bdn., I838-56 (Neudr. I963)

Hist. Zs., I859 ff.

Internationales Arch. für Sozialgesch. der dt. Lit., I976 ff.

Internationales Germanistenlex. I800-I950, hg. u. eingel. C. König, 3 Bde., 2003

Inscape, Ottawa/Kanada I959 ff.

Inventar

JASILO

Jb. Darmstadt

$\mathrm{JbFDH}$

JEGP

Jöcher

Jördens

Killy

Inventar zu den Nachlässen emigrierter dt.sprachiger Wissenschaftler in Arch. $u$. Bibl. der Bundesrepublik Dtl., hg. K.-D. Lehmann, 2 Bde., I993

Jb. des Adalbert-Stifter-Inst. des Landes Oberöst., I994 ff. Dt. Akad. für Sprache u. Dg., Darmstadt, Jb., I953 ff. Jb. des Freien Dt. Hochstifts, I920 ff.

The Journal of English and Germanic Philology, Urbana/Ill. I897 ff.

C. G. Jöcher, Allg.

Gelehrten-Lex., 4 Bde., I750-87

K. H. Jördens, Lex. dt. Dichter u. Prosaisten,

6 Bde., I806-II

Lit. Lex. Autoren u. Werke dt. Sprache, hg. W. Killy, I5 Bde., I988-93 (2., vollständig überarb. Aufl. u.d.T.: Killy Lit.lex. Autoren u. Werke des dt.sprachigen Kulturraumes, hg. W. Kühlmann, I3 Bde., 2008-I2) Krit. Lex. zur dt.sprachigen Gegenwartslit., hg. H. L. Arnold, I978 ff. 
Klingner/Lieb

KLL

$\mathrm{KLL}^{3}$

KNLL

Kraus LD

Kussmaul

LAL

LCI

LE

Lennartz

Lessing Yb.

LeuvBijdr

Lex. dt.-jüd.

Autoren

LexKJugLit

LexMA

LGL

LiLi
J. Klingner/L. Lieb, Hdb.

Minnereden. Mit Beitr. v

I.-E. Dorobaţu u. a., 2 Bde., $2 \mathrm{OI} 3$

Kindlers Lit.-Lex., 7 Bde. u. Erg.Bd., I965-74

Kindlers Lit.-Lex., hg. H. L.

Arnold, 3., völlig neu bearb.

Aufl., I8 Bde., 2009

Kindlers Neues Lit. Lex., hg. W. Jens, 22 Bde.,

I988-98

C. v. Kraus, Dt. Lieder-

dichter des I3. Jh., I Text, I952, II Komm. (besorgt v.

H. Kuhn), I958 (2., v. G.

Kornrumpf durchges. Aufl., 2 Bde., I978)

I. Kussmaul, Die Nachlässe u. Slg. des DLA, 2 Bde., I999

G. Goetzinger, C. D. Conter u. a., Luxemburger Autorenlex., Mersch 2007

Lexikon der christlichen Ikonographie, 8 Bde., Rom u. a. $1968-70$

Das lit. Echo, I898 ff.

F. Lennartz, Dt. Schriftst. des 20. Jh. im Spiegel der Kritik, 3 Bde. u. Reg.Bd., I984

Lessing Yearbook, I969 ff.

Leuvense Bijdragen, Löwen I9IO ff.

Lex. dt.-jüd. Autoren. Redaktionelle Leitung: R.

Heuer, 2I Bde., I992-20I3

Lex. der Kinder- u. Jugendlit., hg. K. Doderer, 3 Bde. u. ErgBd., I975-82

Lex. des MA, 9 Bde., I980-98

Lex. der dt.sprachigen Gegenwartslit. seit I945, begr. v. H. Kunisch, fortgeführt v. H. Wiesner u. a., neu hg. T. Kraft, 2 Bde., 2003

LiLi, Zs. für Lit.-wiss. u. Linguistik, I97I ff.
LitJb

Liliencron

LöstE

LThK

LuK

MAL

Manitius

Marienlex.

Metzler Lit.

Chronik

Meusel

MF

MG

MGG

MGS

MignePL

MIÖG

MLN

MLQ
R. v. Liliencron, Die hist. Volkslieder der Deutschen vom I3. bis zum I6. Jh., 4 Bde. u. Nachtr., I865-69 (Neudr. I966)

Lit.wiss. Jb. der Görresgesellsch., NF, I96I ff.

S. Bolbecher, K. Kaiser, Lex. der öst. Exillit., 2000 Lex. für Theol. u. Kirche, 2. Aufl., ro Bde. u. Reg., I957-67; 3., völlig neu bearb. Aufl., II Bde., I993-200I.

Lit. u. Kritik, I966 ff.

Modern Austrian Literature, Binghamton/N. Y. I968 ff. M. Manitius, Gesch. der lat. Lit. des MA, 3 Tle., I9II-3I Marienlex., hg. R. Bäumer u. L. Scheffczyk, 6 Bde., I988-94

V. Meid, Metzler Lit. Chronik, ${ }^{3} 2006$

J. G. Meusel, Lex. der vom Jahre I750 bis I 800 verstorbenen teutschen Schriftst., I5 Bde., I802-I6

Des Minnesangs Frühling. 38., erneut revidierte Aufl., bearb. v. H. Moser u. H.

Tervooren, I988

Monumenta Germaniae historica inde $\mathrm{ab}$ a. C. 500 usque ad a. I500, I826 ff.

Die Musik in Gesch. u. Gegenwart, hg. F. Blume, I7 Bde., I949-86; 2., neu bearb. Ausg. hg. L. Finscher, Personentl., I7 Bde. u. I Suppl.bd., I999-2008. Michigan Germanic Studies, Ann Arbor/Mich., I975 ff. Patrologiae cursus completus, series latina, hg. J. P. Migne, Paris I844 ff.

Mitt. des Inst. für öst. Gesch.forsch., I880 ff. Modern Language Notes, Baltimore/Maryland I886 ff. Modern Language Quarterly, Seattle/Wash., I940 ff. 
Mommsen

Morvay-Grube

MSD

NDB

$\mathrm{NDH}$

NDL

Neoph.

Neudrucke

Neumeister-

Heiduk

NGS

NHdG

NLit

NM
W. A. Mommsen, Die

Nachlässe in den dt. Arch.

(mit Ergänzungen aus an-

deren Beständen). Bearb.

im Bundearch. in Koblenz,

2 Tle., I97I u. I983 (wird

nach Nrn. zitiert)

Monatshefte (für den dt.

Unterricht, dt. Sprache u.

Lit.), Madison/Wisc. I899 ff.

K. Morvay, D. Grube, Bibliogr. der dt. Predigt des

MA, I974

K. Müllenhoff, W. Scherer (Hg.), Denkmäler Dt.

Poesie u. Prosa aus dem 8 .

bis I2. Jh., I864 (3. Aufl.

bearb. v. E. Steinmeyer, 2

Bde., I892; Neudr. I964)

Internationales Biogr. Arch.

IBA Munzinger-Arch.,

I949 ff.

Gelehrten- u. Schriftsteller-

nachlässe in den Bibl. der

Dt. Demokrat. Republik,

3 Tle., I959-7I (wird nach

Tln. u. Nrn. zitiert)

Neue Dt. Biogr., hg. v. der Hist. Kommission bei der Bayer. Akad. der Wiss., I953 ff.

Neue Dt. Hefte, I954-89

Neue Dt. Lit., I953-2004

Neophilologus, Groningen

I95I ff.

Neudr. dt. Lit.werke des

XVI. u. XVII. Jh., begr. v.

W. Braune, fortgefuihrt $\mathrm{u}$.

hg. v. E. Beutler, I876 ff.

E. Neumeister, De Poetis

Germanicis, hg. F. Heiduk

in Zus.arbeit mit G. Mer-

wald, I978

New German Studies, Hull I973 ff.

Neues Hdb. der dt. Ggw.lit. seit I945, begr. v. H. Ku-

nisch, hg. D.-R. Moser, ak-

tualisierte Ausg. 1993

Die Neue Lit., I93I ff.

Neuphilol. Mitt., Helsinki

I899 ff.
NN

NR

NSR

ÖBL

ÖGL

Öst. Kat.-Lex.

$\mathrm{OL}$

$\mathrm{OM}$

PPB (Halle)

PPB (Tüb.)

PEGS

PMLA

Poetica

PP

PQ

Pyritz

Raabe,

Expressionismus
Neuer Nekrolog der Deutschen, hg. A. Schmidt, B. F. Voigt, 30 Bde., I824-56

(Die) Neue Rundschau, I9O4 ff.

Neue Schweizer Rundschau, I922 ff.

Öst. Biogr. Lex. I8I5-I950, I957 ff.

Öst. in Gesch. u. Lit., I957 ff.

Kat. Lex. zur öst. Lit. des 20. Jh. (hg. G. Ruiss.)

2 Bde., I995

Orbis Litterarum, Kopenhagen I943 ff.

Mitt. des Ver. für Gesch. u. Landeskunde v. Osnabrück, I $848 \mathrm{ff}$.

Beitr. zur Gesch. der dt. Sprache u. Lit., begr. v. H. Paul u. W. Braune, Halle I874 ff. (ab I955: Zusatz «Halle»)

Beitr. zur Gesch. der dt. Sprache u. Lit., Tübingen I955 ff.

Publ. of the English Goethe Society, Leeds I886-I9I2, NS I924 ff.

Publ. of the Modern Language Association of America, Menasha/Wisc. I884 ff.

Poetica. Zs. für Sprach- u. Lit.wiss. Amsterdam I969 ff. Philologica Pragensia, Prag I958 ff.

Philological Quarterly, Iowa City 1922 ff.

Bibliogr. zur dt. Lit.gesch. des Barockzeitalters, begr. v. H. Pyritz, fortgeführt u. hg. v. I. Pyritz, 3 Bde., I985-94

P. Raabe, Die Autoren u. Bücher des lit. Expressionismus. Ein bibliogr. Hdb. In Zus.arbeit mit I. HannichBode, 2., verb. Aufl., I992 
RE

Redlich

Renner

RG

RGG

Riemann

RL

RLC

RLW
Realencyklopädie für protestant. Theol. u. Kirche, hg. A. Hauck, 3. Aufl., 24 Bde., I 896-I9I3

M. Redlich, Lex. dt.balt. Lit. Eine Bibliogr., I989

G. Renner, Die Nachlässe in den Bibl. u. Museen der Republik Öst., I993

Recherches Germaniques, Straßburg I97I ff.

Die Rel. in Gesch. u. Ggw., 3. Aufl., 6 Bde., I957-62; 4., völlig neu bearb. Aufl. u.d.T.: Rel. in Gesch. u. Ggw., 8 Bde., I998-2005

H. Riemann, Musiklex. I2., völlig neubearb. Aufl. in 3 Bdn., hg. W. Gurlitt, H. H. Eggebrecht, Personentl. Bd. I u. 2, I959-6I, ErgBde., hg. C. Dahlhaus, 2 Bde., I972-75; RiemannMusik-Lex., I3. aktualisierte NA, hg. W. Ruf in Verb. mit A. van Dyck-Hemming, 5 Bde., 2012

Reallex. der dt. Lit.gesch., hg. P. Merker u. W.

Stammler, 4 Bde., I925-3I; 2., neubearb. Aufl. hg. W. Kohlschmidt u. W. Mohr, 5 Bde., I955-88

Revue de littérature comparée, Paris I92 I ff.

Reallex. der dt. Lit.wiss. Neubearb. des RL, hg. H. Fricke, J.-D. Müller u. K. Weimar, 3 Bde., I997-2003

Rohnke-Rostalski Lit. Nachlässe in NordrheinWestf. Eine Bestandsaufnahme, bearb. v. D. RohnkeRostalski, hg. E. Niggemann, I995

Repertorium der Sangsprüche $u$. Meisterlieder des I2. bis I8. Jh., hg. H. Brunner, B. Wachinger, I3 Bde. u. 3 RegBde., I986-2009
SAB

SAM

SchillerJb

Schmidt,

Quellenlex.

Schmutz-Pfister

Schottenloher

$\mathrm{SdZ}$

Seminar

SN

Sommervogel

Spalek

Spalek/Hawrylchak J. M. Spalek, S. H. Hawrylchak, Guide to the Archival Materials of the GermanSpeaking Emigration to the United States after I933, 4 Bde., I978-97

SPIEL Siegener Periodicum zur Internationalen Empir. Lit.wiss., I982 ff.

SR Schweizerische Rundschau, I900 ff.

SuF Sinn u. Form, I949 ff.

StudiGerm Studi Germanici, Rom I963 ff. 
Theater-Lex.

Thieme-Becker

TirLit

TRE

TuK

VASILO

VD I7

VL
W. Kosch, Dt. Theater-Lex. Biogr. u. bibliogr. Hdb., I953 ff.

U. Thieme u. F. Becker, Allg. Lex. der bildenden Künstler v. der Antike bis zur Ggw., 37 Bde., I907-50

Lex. Lit. in Tirol, hg. A. Unterkircher u. C. Riccabona (Forschungsinst. Brenner-Arch., Innsbruck; Internet-Edition) $2006 \mathrm{ff}$. Theolog. Realenzyklopädie, 36 Bde., I977-2005

Text + Kritik, 1963 ff.

Adalbert Stifter-Inst. des Landes Oberöst., Vjs., I952-92; Forts. siehe JASILO

VD i6 Verz. der im dt. Sprachraum erschienenen Drucke des I6. Jh., bearb. I. Bezzel, 25 Bde., 1983-2000

Verz. der im dt. Sprachraum erschienenen Drucke des I7. Jh. (nur online unter: http://www.vdi7.de/).

Die dt. Lit. des MA. Verfasserlex., hg. W. Stammler u. K. Langosch, 5 Bde., I933-55; 2., völlig neu bearb. Aufl., hg. K. Ruh u. a., I4 Bde., I977-2008

VL Dt. Hum.

Dt. Humanismus I480-I520. Verfasserlex., hg. F. J.

Worstbrock, 2 Bde., 2005-I3 H. Vollmer, Allg. Lex. der bildenden Künstler des 2o. Jh., 6 Bde., I953-62
Wall

WB

WBN

Westfäl.

Autorenlex.

WirkWort

WSB

Wurzbach

ZDU

Zedler

ZfdA

$\mathrm{ZfdPh}$
R. Wall, Lex. dt.sprachiger Schriftstellerinnen im Exil I933 bis I945 (überarb. u. aktualisierte NA) 2004 Weimarer Beitr., I955 ff. Wolfenbütteler BarockNachr., I974 ff.

Westfäl. Autorenlex. (I: I750-I800, 2: I800-I850, 3: 1850-1900, 4: 1900-1950), hg. u. bearb. v. W. Gödden u. I. Nölle-Hornkamp, I993-2002

G. von Wilpert u. A. Gühring, Erstausgaben dt.

Dg. Eine Bibliogr. zur dt. Lit. I600-I900, 2., vollst. überarb. Aufl., I992

Wirkendes Wort, I950/5I ff. Sb. der Akad. der Wiss. zu Wien, Phil.-hist. Kl., I $848 \mathrm{ff}$

C. v. Wurzbach, Biogr. Lex. des Kaiserthums Öst., 60 Bde., I856-91

Zs. für dt. Unterricht, I887-I9I9

Großes vollständiges Universal-Lex. aller Wiss. u. Künste, 64 Bde. u. 4 Suppl.bde., I732-54, Nachdr. I993 ff.

Zs. für dt. Altertum und dt. Lit., I876 ff. (Zs. für dt. Alterthum, I84I-76)

Zs. für dt. Philol., I869 ff.

$\star=$ geboren $\quad \dagger=$ gestorben $\rightarrow=$ siehe $\quad \sim$ steht unter «Literatur» anstelle des Stichworts

Bei Verweisen auf Artikel in den Ergänzungsbänden wird die Zahl des betreffenden Bandes angegeben; Verweise auf Artikel im Hauptalphabet enthalten diese Angaben nicht. 


\section{VERFASSER-SIGLEN}

BJ Bruno Jahn

CF Christoph Fasbender

$\mathrm{CMO}$ Claus-Michael Ort

$\mathrm{EH}$

FA

Eleonore Hofmann

Florian Altenhöfer
MM

MMü

RBS
Mike Malm

Mario Müller

Reimund B. Sdzuj

Suliko Hofschulte-Mikelashvili 
